\title{
Scaling of hypersonic shock/turbulent boundary layer interactions
}

\author{
Clara M. Helm $\odot$ and M. P. Martín ${ }^{*} *$ \\ Department of Aerospace Engineering, University of Maryland, College Park, Maryland 20742, USA
}

(Received 10 October 2019; accepted 4 November 2020; published 16 July 2021)

A separation length scaling method for shock/turbulent boundary layer interactions (STBLIs) is considered. A modification of the scaling method of Souverein et al. [J. Fluid Mech. 714, 505 (2013)] is introduced to account for heat transfer effects. As a further generalization of the model, a control volume analysis applied to a cylinder-with-flare geometry demonstrates that this axisymmetric geometry scales with the same parameters as the two-dimensional interactions. The current modified scaling is evaluated for a large range of STBLI conditions. A database of STBLI flows at Mach 2-3 has been collected from the available literature and includes both reflected shock and compression ramp data with various wall heat transfer conditions. A new Large Eddy Simulation database of Mach 7 and Mach 10 cold wall compression ramp flows with parametrically varying ramp angle is also utilized. In addition, the Mach 10 compression ramp experiments of Elfstrom [J. Fluid Mech. 53, 113 (1972)] as well as the Mach 10 cylinder-with-flare experiments of Coleman (Ph.D. thesis, University of London, 1973) and Brooks et al. (AIAA Paper No. 2017-3325, 2017) are included in the evaluation. It is shown that this new generalized scaling method results in a linear collapse of all incipiently separated STBLI data. No collapse is observed for fully separated interactions. Arguments for the physical mechanisms affecting the separation length scaling for the two STBLI regimes, incipient and fully separated, that are consistent with the data trends are presented. Namely, for incipient cases, the distribution of momentum in the incoming boundary layer is key to separation length scaling. In contrast, for fully separated cases, we postulate that the presence and strength of inviscid vortical structures significantly affects the separation length. Furthermore, we postulate how the distinct flow dynamics of the boundary layer and of the inviscid vortical structures mechanisms found in STBLI interplay and become dominant over the other.

DOI: 10.1103/PhysRevFluids.6.074607

\section{INTRODUCTION}

Souverein et al. [1] (SBD hereafter) introduced a scaling for the separation length in twodimensional, supersonic shock-separated flows. The scaling is based on mass conservation arguments and depends only on the freestream Mach number, upstream boundary layer displacement thickness, and flow deflection angle. The interaction strength metric is an expression that approximates the ratio of pressure jump across the shock structure to the pressure jump required for the onset of separation and is a function of inviscid pressure ratio and freestream Mach number.

\footnotetext{
*mpmartin@umd.edu
}

Published by the American Physical Society under the terms of the Creative Commons Attribution 4.0 International license. Further distribution of this work must maintain attribution to the author(s) and the published article's title, journal citation, and DOI. 
To test their scaling method, SBD compiled from the literature a large database of shock/turbulent boundary layer interactions (STBLIs) that included experimental and computational data of both reflected shock and compression ramp interactions at various states of separation and Reynolds numbers. The database consisted of interactions with freestream Mach numbers ranging from 1.7 to 5. One of the key features of the SBD scaling is the collapse of both compression ramp and reflected shock data to the same curve.

The data compilation of SBD included predominantly adiabatic shock interactions. Jaunet et al. [2] (JDD hereafter) showed that the SBD scaling method does not account for variations in separation length caused by wall heat transfer. The authors derived an alternate nondimensionalized shock strength metric based on the free interaction theory of Chapman et al. [3]. Using their data of a Mach 2.3 reflected shock configuration with both adiabatic and heated walls, and also the adiabatic and cold wall Mach 3 compression ramp data of Spaid and Frishett [4], JDD demonstrated the effectiveness of their new scaling for collapsing the separation data of STBLIs with different heat transfer conditions. Their results were further corroborated by the reflected shock direct numerical simulation (DNS) at Mach 2.3, of Volpiani et al. [5]. JDD, however, were unable to demonstrate their scaling for any STBLI with Mach number above 3 or for any appreciable range of Reynolds number.

In this paper, we relate the results of JDD to the original scaling of SBD and derive a more general separation-length-to-shock-strength scaling that includes heat transfer effects across all Reynolds numbers and freestream Mach numbers. It is then our task to evaluate the viability and quality of the proposed scaling law. In doing so, we extend any such STBLI separation scaling law into the hypersonic regime by introducing a database of Mach 7 and Mach 10 compression ramp data. This paper is organized as follows. In Sec. II, we use a control volume analysis of an axisymmetric cylinder-flare configuration to demonstrate that this geometry scales by the same relation as the two-dimensional interactions. In Sec. III, we present a modification of the interaction strength metric that accounts for wall heat transfer effects. In Sec. IV, we introduce our database of hypersonic compression ramp STBLI. The scaling modification of Sec. III and the three-dimensional (3D) scaling of Sec. II are then evaluated for hypersonic conditions. The Mach 10 experimental compression ramp data of Elfstrom [6], the Mach 10 cylinder-flare experimental data of Coleman [7], and the recent Mach 10 cylinder-flare data of Brooks et al. [8] are also included in the evaluation. A discussion of the results is provided in Sec. V.

\section{SEPARATION LENGTH SCALING AND THE GENERALIZATION TO AN AXISYMMETRIC GEOMETRY}

The expression for the nondimensionalized separation length $L^{*}$ as derived by SBD is

$$
L^{*} \equiv\left(\frac{\dot{m}_{\text {post }}^{*}}{\dot{m}_{\text {pre }}^{*}}-1\right)=\frac{L}{\delta^{*}} G_{3}\left(M_{\mathrm{e}}, \phi\right) .
$$

Here $L^{*}$ is by definition the "mass deficit ratio," the term inside the brackets of Eq. (1), which includes the ratio of the outgoing boundary layer deficit of mass flux $\dot{m}_{\text {post }}^{*}=\rho U \delta_{\text {post }}^{*}$ to the incoming boundary layer deficit of mass flux $\dot{m}_{\text {pre }}^{*}=\rho U \delta_{\text {pre }}^{*}$ as determined by a control volume analysis of the interaction region. For the compression ramp configuration, SBD define the dimensional separation length $L$ as the distance between the mean separation shock foot and the corner. For the reflected shock configuration, $L$ is the distance between the points of intersection at the wall of the mean impinging shock and the mean reflected shock. The function $G_{3}$ is a scaling factor based on the flow deflection angle $\phi$ and the inviscid shock angle $\beta$. Although $G_{3}$ is theoretically dependent on the interaction geometry, SBD arrived at the same expression for both the compression ramp and the reflected shock two-dimensional flow configurations:

$$
G_{3}\left(M_{\mathrm{e}}, \phi\right)=\frac{\sin (\beta) \sin (\phi)}{\sin (\beta-\phi)} .
$$




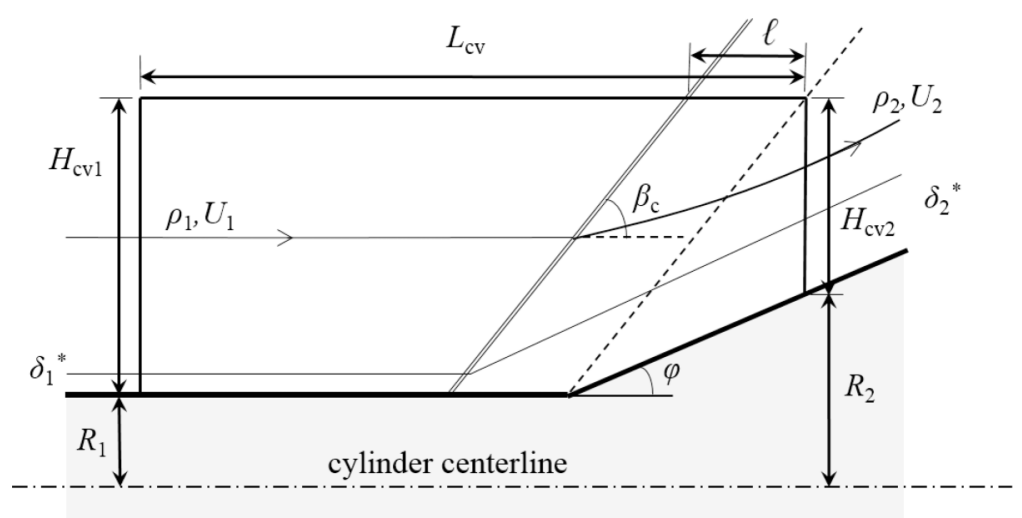

FIG. 1. Control volume for the cylinder-flare configuration. The notation of Souverein et al. is adopted here (see Figure 5 in [1]).

We now derive the separation length scaling for an axisymmetric cylinder-flare STBLI geometry. The control volume for the cylinder flare in Fig. 1 is similar to that used by SBD for the twodimensional compression ramp case; however, for this case we introduce the cylinder radii at the control volume inlet and outlet. We have adopted the same notation for the control volume as SBD to facilitate the comparison of the current analysis with their original formulation. The control volume is also assumed to sweep the full $360^{\circ}$ around the centerline axis. For this derivation we assume that any three-dimensional relief effects along the flare are such that the variation in the wall-normal flow profiles (e.g., $U_{2}, \rho_{2}$, etc.) are minimal and can be approximated by a uniform flow. For example, in the computational solutions by Sims [9] of an inviscid conical shock with cone angle of $30^{\circ}$ and freestream Mach number of 10, the variation in the postshock similarity profiles was less than $0.5 \%$ for wall-parallel velocity and less than $5 \%$ for density. In Fig. 1, the cylinder-flare shock angle is indicated by $\beta_{\mathrm{c}}$ to distinguish it from the two-dimensional oblique shock solution angle $\beta$ occurring at the same freestream Mach number and flow deflection angle.

If the inviscid flow around the flare is considered, conservation of mass over the control volume in Fig. 1 results in the relation

$$
\rho_{1} U_{1}\left[\left(H_{\mathrm{cv} 1}+R_{1}\right)^{2}-R_{1}^{2}\right]-\frac{\rho_{2} U_{2}}{\cos (\phi)}\left[\left(H_{\mathrm{cv} 2}+R_{2}\right)^{2}-R_{2}^{2}\right]=0 .
$$

For the viscous flow with the turbulent boundary layer displacement thickness $\delta^{*}$ and the shock offset distance $\ell$, conservation of mass for the same control volume gives

$$
\begin{aligned}
& \rho_{1} U_{1}\left[\left(H_{\mathrm{cv} 1}+R_{1}\right)^{2}-\left(R_{1}+\delta_{1}^{*}\right)^{2}\right]-\frac{\rho_{2} U_{2}}{\cos (\phi)}\left[\left(H_{\mathrm{cv} 2}+R_{2}\right)^{2}-\left(R_{2}+\frac{\delta_{2}^{*}}{\cos (\phi)}\right)^{2}\right] \\
& -2 \ell \rho_{2} U_{2} \sin (\phi)\left(R_{2}+H_{\mathrm{cv} 2}\right)=0 .
\end{aligned}
$$

Subtracting Eq. (3) from Eq. (4) gives

$$
\rho_{1} U_{1}\left[\left(R_{1}+\delta_{1}^{*}\right)^{2}-R_{1}^{2}\right]-\frac{\rho_{2} U_{2}}{\cos (\phi)}\left[\left(R_{2}+\frac{\delta_{2}^{*}}{\cos (\phi)}\right)^{2}-R_{2}^{2}\right]-2 \ell \rho_{2} U_{2} \sin (\phi)\left(R_{2}+H_{\mathrm{cv} 2}\right)=0,
$$

which can be rearranged to solve for $\ell$ as

$$
\ell=\frac{\frac{\rho_{2} U_{2}}{\cos ^{2}(\phi)}\left[2 R_{2} \delta_{2}^{*}+\frac{\delta_{2}^{* 2}}{\cos (\phi)}\right]-\rho_{1} U_{1}\left[2 R_{1} \delta_{1}^{*}+\delta_{1}^{* 2}\right]}{2 \rho_{2} U_{2} \sin (\phi)\left(R_{2}+H_{\mathrm{cv} 2}\right)} .
$$




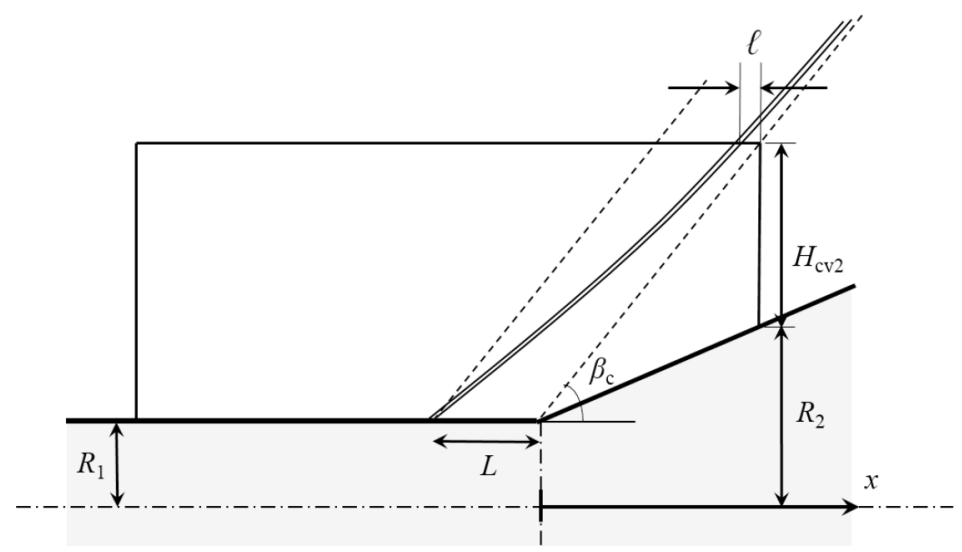

FIG. 2. Diagram of the cylinder-flare shock position showing the difference between $\ell$ defined for the control volume and the actual flow separation length $L$.

To simplify Eq. (6) it is assumed that the control volume is such that $\delta_{1}^{*} \ll R_{1}$ and $\delta_{2}^{*} \ll R_{2}$ resulting in the expression

$$
\frac{\ell}{\delta_{1}^{*}}=\left[\frac{\delta_{2}^{*}}{\delta_{1}^{*}}\left(\frac{R_{2}}{\cos ^{2}(\phi)}\right)-\frac{\rho_{1} U_{1}}{\rho_{2} U_{2}}\left(R_{1}\right)\right] \frac{1}{\sin (\phi)\left(R_{2}+H_{\mathrm{cv} 2}\right)} .
$$

Finally, by using the continuity relation across the shock such that $U_{1} \rho_{1} \sin \left(\beta_{\mathrm{c}}\right)=U_{2} \rho_{2} \sin \left(\beta_{\mathrm{c}}-\phi\right)$, Eq. (7) can be rewritten as

$$
\frac{\ell}{\delta_{1}^{*}}=\left[\frac{\rho_{2} \frac{U_{2}}{\cos (\phi)} \frac{\delta_{2}^{*}}{\cos (\phi)}}{\rho_{1} U_{1} \delta_{1}^{*}}\left(\frac{2 \pi R_{2}}{2 \pi R_{1}}\right)-1\right] \frac{\sin \left(\beta_{\mathrm{c}}-\phi\right)}{\sin \left(\beta_{\mathrm{c}}\right) \sin (\phi)} \frac{R_{1}}{R_{2}+H_{\mathrm{cv} 2}} .
$$

By comparing Eq. (8) with Eq. (1), the term in the square brackets is easily recognized as the mass deficit ratio $\left(\dot{m}_{\text {post }}^{*} / \dot{m}_{\text {pre }}^{*}-1\right)$ for the cylinder cross section. The inverse of the two-dimensional flow deflection function $G_{3}$ also appears in Eq. (8) and is multiplied by the ratio $R_{1} /\left(R_{2}+H_{\mathrm{cv} 2}\right)$. Since $H_{\mathrm{cv} 2}$ and $R_{2}$ do not cancel out in the derivation of Eq. (8), it appears that the separation length is dependent on the choice of control volume. This does not make sense physically seeing as a given STBLI flow will have a specific mean separation length $L$ regardless of the choice of control volume. This apparent problem in the derivation can be remedied with the following reasoning. In Fig. 1, $l$ is defined as the distance between the crossing point of the shock at the top boundary and the top right corner of the control volume. It is assumed that the shock remains parallel to the inviscid shock inside the control volume, thus making $\ell$ equivalent to the actual separation length $L$. This is approximately true for the two-dimensional ramp but not for the cylinder flare. For instance, if the distance $R_{2}+H_{\mathrm{cv} 2}$ is rewritten in terms of $R_{1}, \beta_{\mathrm{c}}$, and a coordinate variable $x$ defined as the distance along the cylinder centerline from the flare corner (see Fig. 2), Eq. (8) becomes

$$
\frac{\ell}{\delta_{1}^{*}}=\left(\frac{\dot{m}_{\mathrm{post}}^{*}}{\dot{m}_{\mathrm{pre}}^{*}}-1\right) G_{3}^{-1} \frac{R_{1}}{R_{1}+x \tan \left(\beta_{\mathrm{c}}\right)} .
$$

In this form one can clearly see that the length $\ell$ goes to 0 as $x$ increases indefinitely. It would appear then that the actual separation length $L$ is determined from the limit of Eq. (9) as $x$ goes to 0 which ultimately results in Eq. (1). By this analysis, the cylinder-flare configuration scales by the same relation as the two-dimensional compression ramp. Any three-dimensional effects are therefore entirely contained in the difference in shock angle $\beta_{\mathrm{c}}$ and downstream-to-upstream pressure ratio generated by the flared geometry as compared to the two-dimensional ramp. Note also from Eq. (8) that, for a given angle $\phi$ and control volume dimensions, $R_{2}$ is equal to $R_{1}$ plus a constant. If $R_{1}$ 
goes to infinity, which is the equivalent of a flat plate, the ratio $R_{2} / R_{1}$ goes to 1 , which results in the original two-dimensional ramp expression of Eqs. (1) and (2). This further implies that the shock angle $\beta_{\mathrm{c}}$ is a function of the cylinder radius $R_{1}$ and will in fact vary between the oblique shock angle $\left(R_{1}=\infty\right)$ and the conical shock angle $\left(R_{1}=0\right)$.

\section{INTERACTION STRENGTH SCALING WITH HEAT TRANSFER}

It was proposed by SBD that the correct interaction strength metric for the scaling of the nondimensionalized separation length data is the ratio of pressure jump across the interaction $\Delta P$

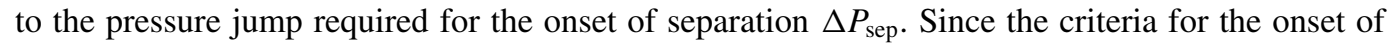
separation is not typically known for a given STBLI flow, SBD derived the interaction strength parameter $S e_{\mathrm{SBD}}^{*}$ as an approximation of $\Delta P / \Delta P_{\text {sep. }}$. The assumption was made that $\Delta P_{\text {sep }}$ scales as the dynamic pressure $q_{\mathrm{e}}$ so that

$$
S e_{\mathrm{SBD}}^{*}=k \frac{\Delta P}{q_{\mathrm{e}}} .
$$

The normalization constant $k$ is introduced to ensure $S e_{\mathrm{SBD}}^{*} \approx 1$ at the onset of separation and is assumed to be independent of the freestream Mach number. From a compilation of experimental data for which $\Delta P_{\text {sep }}$ was known, SBD showed that $k=2.5$ and is also independent of Reynolds number at least up to $\operatorname{Re}_{\theta}=3 \times 10^{5}$ [10]. Together with $L^{*}$ from Eq. (1), $S e_{\mathrm{SBD}}^{*}$ was shown to collapse adiabatic data for $M_{\mathrm{e}}$ from 1.7 to 5 and for $\operatorname{Re}_{\theta}$ between $2.3 \times 10^{3}$ and $3 \times 10^{5}$. Reflected shock and compression ramp data, both experimental and computational, were included in the database.

More recently, Jaunet et al. [2] used reflected shock experiments at $M_{\mathrm{e}}=2.3$ with varying deflection angle for both an adiabatic and a heated wall $\left(T_{\mathrm{w}} / T_{\mathrm{r}}=1.9\right.$ where $T_{\mathrm{r}}$ is the adiabatic recovery temperature) to show that the SBD separation strength metric does not collapse the data of STBLIs with varying wall temperature conditions. Based on the separation plateau pressure scaling in the free-interaction theory of Chapman et al. [3], JDD proposed a new shock strength metric defined as

$$
S e_{\mathrm{JDD}}^{*}=\frac{\Delta P}{k_{2} q_{\mathrm{e}} \sqrt{\frac{2 C_{f_{\mathrm{o}}}}{\left(M_{\mathrm{e}}^{2}-1\right)^{1 / 2}}}} .
$$

The denominator of Eq. (11) is the approximation of $\Delta P_{\text {sep }}$ which is now assumed to scale with the incoming boundary layer skin friction coefficients $C_{f o}, M_{\mathrm{e}}$, and $q_{\mathrm{e}}$. The constant $k_{2}$ is again a normalization constant that is introduced so that $S e_{\mathrm{JDD}}^{*} \approx 1$ at the onset of separation. JDD determined from the incident shock angle required to separate their adiabatic boundary layer that $k_{2}=7.14$ for their data. The dependence of $k_{2}$ with $\operatorname{Re}_{\theta}$ or $M_{\mathrm{e}}$ is otherwise unknown.

When $S e_{\text {JDD }}^{*}$ is applied to their reflected shock data together with the adiabatic and cold wall compression ramp data of Spaid and Frishett [4], a much better collapse of $L^{*}$ is achieved as compared to $S e_{\mathrm{SBD}}^{*}$. Because the data of Spaid and Frishett is of similar Mach number and Reynolds number, the same $k_{2}$ was used throughout. In addition, Volpiani et al. [5] also tested the JDD scaling with their DNS database of reflected shock interactions, also at similar freestream conditions, with satisfactory results. Their DNS database included wall temperature ratios $T_{\mathrm{w}} / T_{\mathrm{r}}=1.0,0.5$, and 1.9. The greatest drawback to the scaling of JDD, however, is the lack of knowledge of the dependence of $k_{2}$ on the conditions of any given STBLI flow.

We now propose a scaling of the interaction strength that is based on the combined results of JDD and SBD. For the derivation, two observations are made. First, we note that the SBD scaling was shown to work well for a large range of adiabatic STBLIs. Second, the parameters $S e_{\mathrm{SBD}}^{*}$ and $S e_{\mathrm{JDD}}^{*}$ have nearly the same form in that $S e_{\mathrm{JDD}}^{*}$ is essentially a correction of the SBD normalization constant $k$. We make the assumption that both scaling methods are equivalent for adiabatic interactions and 
(a)

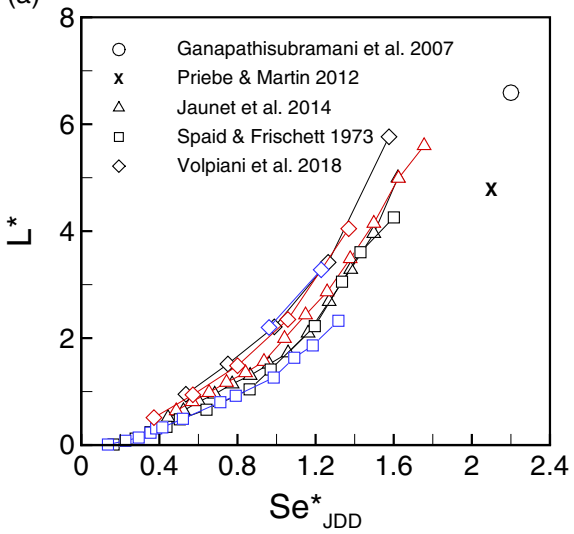

(b)

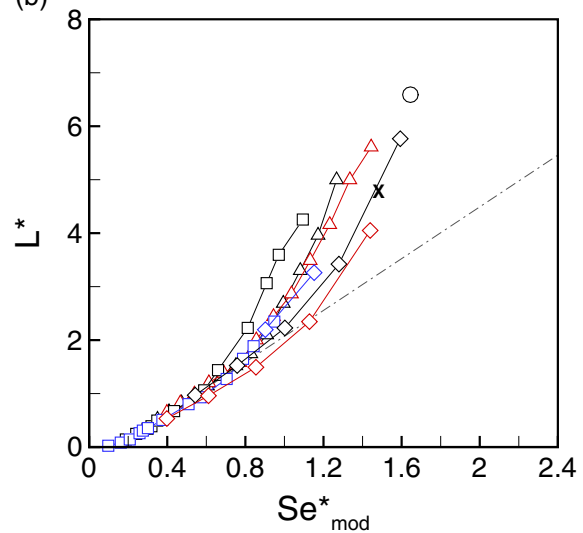

FIG. 3. Compilation of Mach 2-3 STBLI data with various heat transfer conditions scaled by $S e_{\text {JDD }}^{*}$ (a) and again by the $S e_{\text {mod }}^{*}$ (b). Symbol color indicates the wall temperature condition. Black data points are adiabatic walls, red are heated walls, and blue are cold walls.

that $k$ and $k_{2}$ can therefore be related to each other by the following expression.

$$
\frac{1}{k}=k_{2} \sqrt{\frac{2 C_{f \mathrm{o}, \mathrm{a}}}{\left(M_{\mathrm{e}}^{2}-1\right)^{1 / 2}}} .
$$

Here $C_{f o, a}$ is the skin friction coefficient for an adiabatic boundary layer with the same freestream conditions and Reynolds number. If we assume that $k_{2}$ can be determined from the $\Delta P_{\text {sep }}$ of the adiabatic boundary layer, then we arrive at an expression for a modified proportionality constant $k_{\text {mod }}$ that is generalized for any wall temperature condition

$$
k_{\mathrm{mod}}=k \sqrt{\frac{C_{f \mathrm{o}, \mathrm{a}}}{C_{f \mathrm{o}}}} .
$$

The $k$ of SBD is therefore simply scaled by the square root of the ratio of the adiabatic $C_{f \mathrm{o}, \mathrm{a}}$ to $C_{f \mathrm{o}}$ with heat transfer. The modified separation strength metric then becomes

$$
S e_{\text {mod }}^{*}=k_{\mathrm{mod}} \frac{\Delta P}{q_{\mathrm{e}}}=k \sqrt{\frac{C_{f_{\mathrm{o}, \mathrm{a}}}}{C_{f_{\mathrm{o}}}}} \frac{\Delta P}{q_{\mathrm{e}}} .
$$

Even if unknown, $C_{f_{\mathrm{o}, \mathrm{a}}}$ can be estimated using an appropriate skin friction prediction method such as those reviewed by Hopkins and Inouye [11]. It is interesting to mention that if the relation between $k$ and $k_{2}$ of Eq. (12) is used to back out $k_{2}$ for the experiments of JDD, a value of 7.41 is obtained compared to their experimentally determined value of 7.14.

We first test the scaling of $L^{*}$ with $S e_{\text {mod }}^{*}$ on the data of JDD, Spaid and Frishett, and Volpiani et al. We also include the DNS compression ramp of Priebe and Martin [12] with $M_{\mathrm{e}}=2.9$, ramp angle $=$ $24^{\circ}, T_{\mathrm{w}} / T_{\mathrm{r}}=1.0$, and $\operatorname{Re}_{\theta}=2400$, and the experimental compression ramp of Ganapathisubramani et al. [13] with $M_{\mathrm{e}}=2$, ramp angle $\phi=20^{\circ}, T_{\mathrm{w}} / T_{\mathrm{r}}=1.0$, and $\operatorname{Re}_{\theta}=35000$. Figure 3(a) shows all $L^{*}$ data scaled by $S e_{\mathrm{JDD}}^{*}$ as reproduced from Fig. 10 of Jaunet et al. [2] and Fig. 13(b) of Volpiani et al. [5]. Again, the same $k=7.14$ is used for the Priebe and Martin and Ganapathisubramani data points. The data are then rescaled using $S e_{\text {mod }}^{*}$ of Eq. (14) and plotted in Fig. 3(b). Two observations are immediately apparent from this comparison. First, a much closer data collapse occurs for $S e_{\text {mod }}^{*} \lesssim 1$ and the points in this range clearly fall on a linear trend. Second, there is essentially no collapse of the fully separated STBLI data points when scaled by $S e_{\text {mod }}^{*}$. It will be 
TABLE I. Database of hypersonic compression ramp STBLI: flow conditions.

\begin{tabular}{lcccrrrrrr}
\hline \hline Case & Type & $M_{\mathrm{e}}$ & $T_{\mathrm{w}} / T_{\mathrm{r}}$ & $\begin{array}{c}\delta \\
(\mathrm{mm})\end{array}$ & $\begin{array}{c}\delta^{*} \\
(\mathrm{~mm})\end{array}$ & $\operatorname{Re}_{\theta}$ & $\mathrm{Re}_{\tau}$ & $\phi$ & Reference \\
\hline M7 & LES & 7.17 & 0.5 & 4.9 & 2.5 & 3720 & 210 & $8^{\circ}-33^{\circ}$ & Helm and Martin [19] \\
M10 & LES & 9.58 & 0.3 & 18.5 & 13.2 & 7940 & 460 & $15^{\circ}-34^{\circ}$ & Helm and Martin [21] \\
M10w & LES & 9.05 & 0.3 & 19.7 & 11.3 & 8280 & 520 & $34^{\circ}$ & Helm and Martin [19] \\
M10-Elf1 & Exp. & 9.22 & 0.3 & 7.2 & 3.6 & 9010 & 680 & $15^{\circ}-38^{\circ}$ & Elfstrom [6] \\
M10-Elf2 & Exp. & 8.95 & 0.3 & 7.6 & 2.4 & 2900 & 220 & $15^{\circ}-38^{\circ}$ & Elfstrom [6] \\
M10-Col1 & Exp. & 9.22 & 0.3 & 4.6 & 2.3 & 4800 & 390 & $15^{\circ}-40^{\circ}$ flare & Coleman [7] \\
M10-Col2 & Exp. & 8.95 & 0.3 & 9.1 & 5.1 & 2900 & 240 & $15^{\circ}-40^{\circ}$ flare & Coleman [7] \\
M10-CF & Exp. & 9.87 & 0.3 & 23.3 & 12.5 & 8346 & 706 & $34^{\circ}$ flare & Brooks et al. [8] \\
\hline \hline
\end{tabular}

shown in the next section that similar results occur with the hypersonic data. Further discussion of these observations is provided in Sec. V.

\section{THE SCALING OF A HYPERSONIC STBLI DATABASE}

A brief overview of the computational method used to generate our hypersonic STBLI data is given here. The Large Eddy Simulation (LES) solves the Favre-filtered generalized curvilinear Navier-Stokes equations in conserved form. A fourth-order linearly and nonlinearly optimized Weighted Essentially Non-Oscillatory (WENO) method [14,15] is used for the discretization of the inviscid terms. A fourth-order central scheme is used for the discretization of the viscous terms and a low-storage third-order Runge-Kutta method [16] is used for the advancement in time. The Favre-filtered operation results in the addition of several sub-grid-scale (SGS) terms to the set of equations. The SGS shear stress tensor and the SGS heat flux tensor are modeled using the one-coefficient dynamic mixed model in Martin et al. [17]. The SGS turbulent diffusion term is modeled using the velocity-shear stress tensor correlation approximation of Knight et al. [18]. This LES method and SGS models have been validated for the accurate simulation of attached and fully separated hypersonic STBLI by Helm and Martin [19].

Our LES database of hypersonic compression ramp flows consists of a Mach 7 cold wall turbulent boundary layer over ramp angles from $8^{\circ}$ to $33^{\circ}$ and a Mach 10 cold wall turbulent boundary layer over ramp angles from $15^{\circ}$ to $34^{\circ}$. Freestream Mach number and incoming boundary layer properties are listed in Table I together with those from experimental data at similar conditions to the Mach 10 LES. The conditions at both Mach 7 and 10 are low enthalpy flows typical of ground test facilities, and all simulations presented here are nonreacting with constant specific heats and the ideal gas law is assumed to hold. The range of compression ramp angles $\phi$ for each of the incoming flows is also included in Table I. Details of the computational grids are summarized in Table II. Computational domain sizes are given in terms of a reference boundary layer thickness $\delta_{\mathrm{o}}$. The dimension $L_{\mathrm{corner}}$ is the distance from the inlet to the corner of the ramp and $L_{\text {ramp }}$ is the length along the ramp surface measured from the corner to the outlet plane. Grid resolutions are given in units of the inner length

TABLE II. STBLI LES computational grid.

\begin{tabular}{lrccccccr}
\hline \hline Case & $\begin{array}{c}\delta_{\mathrm{o}} \\
(\mathrm{mm})\end{array}$ & $L_{\text {corner }} / \delta_{\mathrm{o}}$ & $L_{\text {ramp }} / \delta_{\mathrm{o}}$ & $L_{\mathrm{y}} / \delta_{\mathrm{o}}$ & $L_{\mathrm{z}} / \delta_{\mathrm{o}}$ & $\Delta x^{+}$ & $\Delta y^{+}$ & $z_{2}{ }^{+}$ \\
\hline M7 & 5.0 & 12 & 12 & 10 & 8 & $25.9-11.2$ & 8.2 & 0.22 \\
M10 & 18.0 & 13 & 8 & 3 & 7 & $30.7-10.0$ & 6.7 & 0.19 \\
M10w & 18.0 & 13 & 12 & 10 & 7 & $26.2-10.7$ & 6.7 & 0.19 \\
\hline \hline
\end{tabular}


TABLE III. Mean flow separation.

\begin{tabular}{|c|c|c|c|c|}
\hline Case & $\phi$ & $L / \delta_{\mathrm{o}}$ & $\Delta t U_{\mathrm{e}} / \delta_{\mathrm{o}}$ & $\Delta t U_{\mathrm{e}} / L$ \\
\hline M7-08 & $8^{\circ}$ & 0 & 150 & \\
\hline M7-17 & $17^{\circ}$ & 0.27 & 500 & 1850 \\
\hline M7-20 & $20^{\circ}$ & 0.49 & 610 & 1240 \\
\hline M7-24 & $24^{\circ}$ & 0.87 & 460 & 530 \\
\hline M7-28 & $28^{\circ}$ & 1.51 & 540 & 360 \\
\hline M7-31 & $31^{\circ}$ & 2.94 & 770 & 260 \\
\hline M7-33 & $33^{\circ}$ & 4.42 & 1970 & 450 \\
\hline M10-15 & $15^{\circ}$ & 0.06 & 140 & 2330 \\
\hline M10-22 & $22^{\circ}$ & 0.27 & 150 & 560 \\
\hline M10-24 & $24^{\circ}$ & 0.40 & 200 & 500 \\
\hline M10-27 & $27^{\circ}$ & 0.59 & 190 & 320 \\
\hline M10-31 & $31^{\circ}$ & 1.10 & 180 & 160 \\
\hline M10-33 & $33^{\circ}$ & 2.28 & 210 & 90 \\
\hline M10-34 & $34^{\circ}$ & 3.10 & 220 & 70 \\
\hline M10w & $34^{\circ}$ & 3.32 & 310 & 90 \\
\hline
\end{tabular}

${ }^{\mathrm{a}}$ Reproduced from Helm and Martin [19].

${ }^{\mathrm{b}}$ Reproduced from Helm and Martin [21].

scale $z_{\tau}$ as indicated by the "+" superscript. A single wide-domain Mach $10,34^{\circ}$ ramp is also included in the database and is listed as case M10w in Tables I and II. The spanwise width of the M10 computational grid is $L_{y}=3 \delta_{\mathrm{o}}$ compared to $10 \delta_{o}$ for both the M7 and M10w runs. We chose to use the narrower grid to run parametric ramp angles at the Mach 10 conditions simply because of the lower computational cost. This is especially true for the higher Reynolds number of the Mach 10 boundary layer compared to the Mach 7 for which $L_{\mathrm{y}}=10 \delta_{\mathrm{o}}$ was used for the parametric runs. As will be discussed in Sec. V, the narrow grid does tend to restrict the spanwise periodicity in the downstream flow of the separated cases; however, the effect that this has on the averaged separation length is small and the same conclusions on the scaling analysis can be made.

The mean separation length $L$ in units of $\delta_{\mathrm{o}}$ is listed in Table III for each LES run. The duration over which the mean field was averaged is also listed in Table III in time units nondimensionalized by both $\delta_{\mathrm{o}}$ and $L$ with freestream velocity $U_{\mathrm{e}}$. Considering that the Strouhal number of the lowfrequency unsteadiness of separated STBLI is typically centered at $\mathrm{St}_{\mathrm{L}}=f L / U_{\mathrm{e}}=0.03$ (Dupont et al. [20] and Priebe and Martin [12] among others), one aperiodic cycle is on the order of $30 L / U_{\mathrm{e}}$ in time. The M10-33 run resolves approximately three low-frequency cycles and M10-34 only two; however, this is sufficient to determine the mean separation length with enough accuracy for the evaluation of the scaling law. See the discussion on the convergence of the mean separation length in Helm and Martin [21]. The mean skin friction distributions and the mean wall pressure distributions for the Mach 7 interactions are shown in Fig. 4. The Mach 10 interactions are shown in Fig. 5. Dashed lines indicate attached and incipiently separated ramp angles and solid lines indicate fully separated ramp angles.

We now apply the scaling using Eqs. (1) and (14) to the data of Table III. The experimental data included in the scaling analysis are the Mach 10 compression ramp data of Elfstrom [6], the Mach 10 cylinder with flare data of Coleman [7] at similar conditions to the compression ramps of Elfstrom, and the Arnold Engineering Development Center (AEDC) cylinder-flare experiment of Brooks et al. [8]. Not all of the information needed to scale the experimental data was available from the respective references. The displacement thickness for the data of Elfstrom was not reported explicitly. We used the boundary layer velocity profile data available from the Ph.D. thesis of Elfstrom [22] together with the Crocco relation for mean velocity and mean temperature to reproduce the experimental profiles of $\left(\rho_{\mathrm{e}} U_{\mathrm{e}}\right)$ from which $\delta^{*}$ could be integrated. Neither the displacement 
(a)

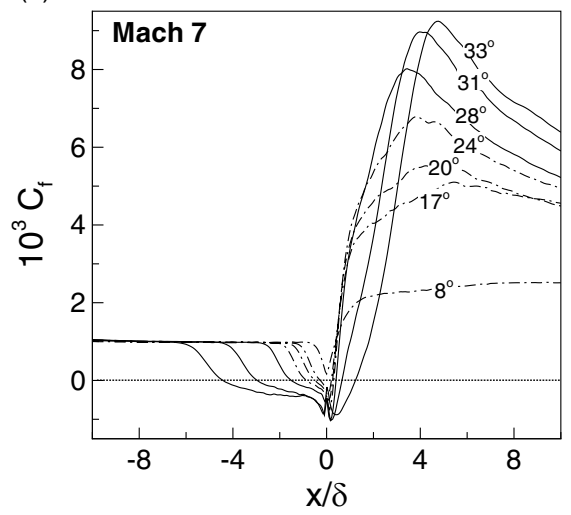

(b)

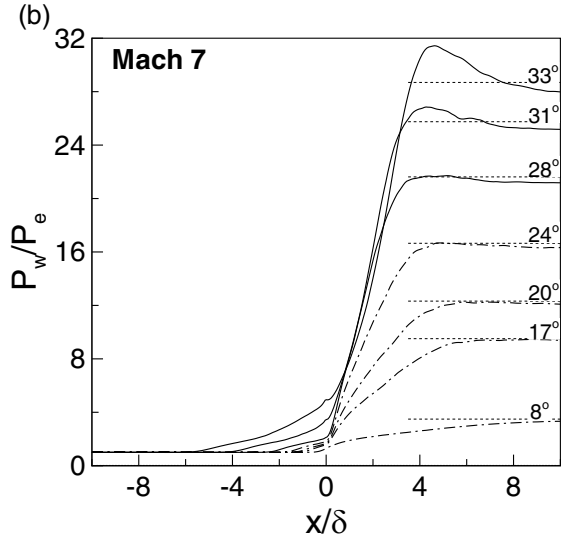

FIG. 4. Skin friction (a) and wall pressure (b) distributions for M7 LES data. Dashed lines indicate attached and incipient separated ramp angles. Solid lines are fully separated ramp angles.

thickness nor the velocity profiles were available for the data of Coleman. Since the data of Coleman and Elfstrom were run in the same experimental facility at the same nominal freestream and wall temperature conditions, we assume here that the ratio $\delta^{*} / \delta$ is the same between M10-Coll and M10-Elf1 and between M10-Col2 and M10-Elf2. The separation lengths $L$ were obtained from the available static pressure distributions [Figs. 16, 17, and 20 of Elfstrom [22]; and Figs. 50(a) and 51(a) of Coleman [7]]. The separation length of the M10-CF was estimated as $L=0.3 \delta$ from the Particle Image Velocimetry mean streamwise velocity field at the flare corner (Fig. 12 in Brooks et al. [8]). The surface pressure measurements of Coleman showed that the pressure on the flare downstream of reattachment approached the conical shock inviscid pressure for all flare angles. Surface pressure data was not available for the AEDC experiment. Inviscid conical shock theory was used to estimate $\beta_{\mathrm{c}}$ and $P_{2} / P_{1}$ for all flare data in Table I. The van Driest II theory [23] was used to determine the adiabatic skin friction coefficient $C_{f \mathrm{o}, \mathrm{a}}$ for the calculation of $k_{\mathrm{mod}}$ for all data. The van Driest II theory was shown by Duan et al. [24,25] to be accurate within 5\% error for Mach numbers up to 12 and wall temperatures $T_{\mathrm{w}} / T_{\mathrm{r}}$ from 1.0 to 0.2 .

The scaling results are plotted in Fig. 6. The incipiently separated interactions, including the cylinder-flare data, appear to be well described by the same linear trend observed in the supersonic

(a)

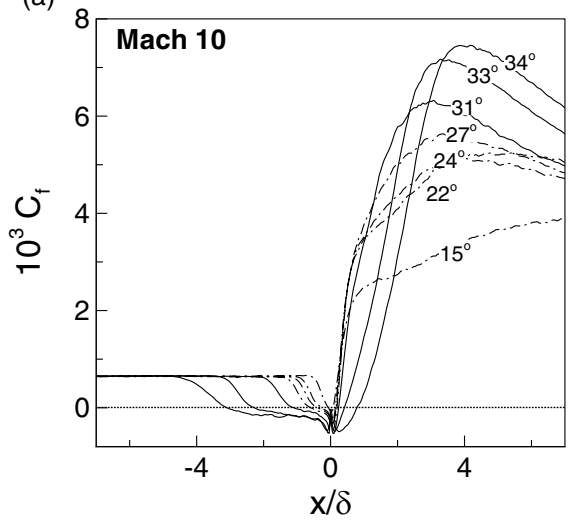

(b)

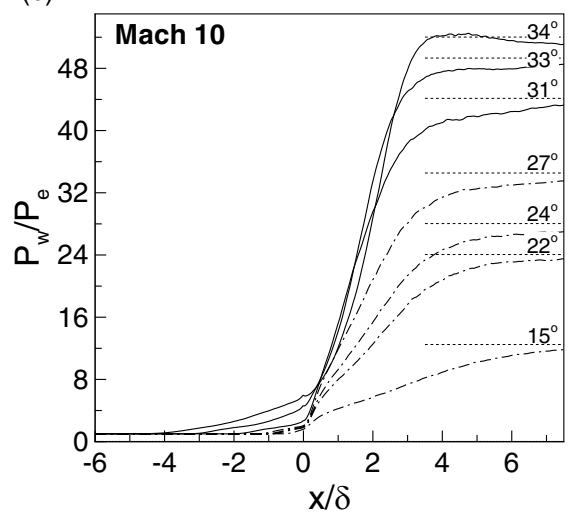

FIG. 5. Skin friction (a) and wall pressure (b) distributions for M10 LES data. Dashed lines indicate attached and incipient separated ramp angles. Solid lines are fully separated ramp angles. 


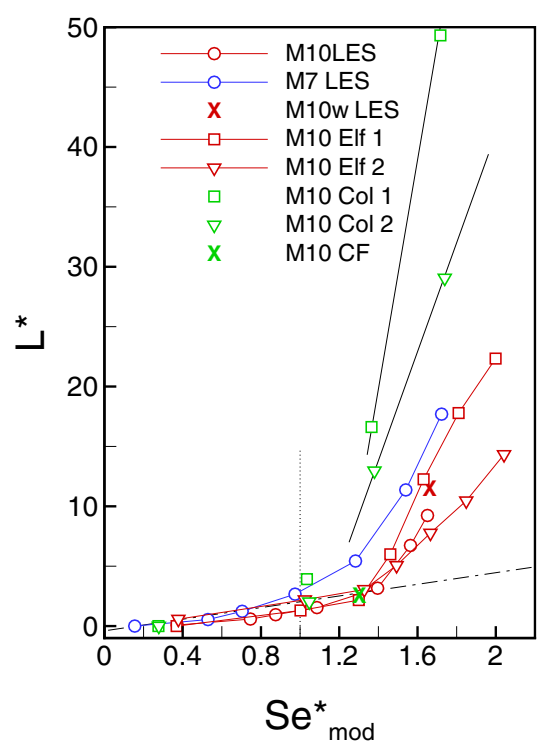

FIG. 6. Separation scaling data of the hypersonic STBLI database of Table I. The dashed line is the linear trend reproduced from Fig. 3(b).

data. As with the supersonic data, significant spreading occurs in the fully separated regime and the scaling law does not hold. The M10, M10w, and M10-Elf1 data are of similar conditions $\left(\operatorname{Re}_{\theta}\right.$, $M_{\mathrm{e}}, T_{\mathrm{w}} / T_{\mathrm{r}}$ ) and these nearly coincide across all ramp angles. The fully separated M10-31, M10-33, and M10-34 are a bit below the M10-Elf1 and M10w data points; however, this is interpreted as a consequence of the narrow computational domain of M10 as will be discussed in the following section. The fully separated cylinder with flare M10-Col1, also at similar conditions as M10-Elf1 but with half the $\operatorname{Re}_{\theta}$, has dramatically larger $L^{*}$ than the compression ramp.

\section{DISCUSSION OF CONCLUSIONS}

All $S e_{\text {mod }}^{*}$ and $L^{*}$ data from Secs. III and IV are plotted together in Fig. 7(a). Both the supersonic and hypersonic data show that there are two distinct linear regions in the curve of $L^{*}$ versus $S e^{*}$ when multiple deflection angles are plotted for the same incoming boundary layer. The point at which the two linear regions intersect has been determined to coincide with the onset of separation for a given boundary layer [1,2]. In corroboration with this assumption, careful observation of the current LES data reveals that the first point on the fully separated branch of each of the M7 and M10 curves (M7-28 and M10-31, respectively) is also the smallest ramp angle simulated for which the spanwise averaged flow field does not instantaneously reattach. A characteristic of these two slopes, which is most apparent in the hypersonic data but is also true for the supersonic data, is that they are not proportional to each other in the same ratio across all data. It is therefore not possible to collapse both the incipiently separated data and the fully separated data simultaneously by using a single proportionality constant such as $k$ in the definition of the shock strength metric. We also point out that this problem of the disproportionality of the slopes remains even if $L^{*}$ is plotted versus $\Delta P / \Delta P_{\text {sep }}$ as is done in Fig. 7(b) for all data for which $\Delta P_{\text {sep }}$ is known. Recall that $S e_{\text {mod }}^{*}$ is an approximation of the ratio $\Delta P / \Delta P_{\text {sep }}$ such that $q_{e} / k_{\bmod } \approx \Delta P_{\text {sep. }}$. In conclusion, it seems that the two branches of incipiently separated and fully separated STBLI are each governed by different physical mechanisms and therefore different scaling laws, each potentially with its own dependence on Reynolds number, Mach number, wall temperature, and geometry. 
(a)

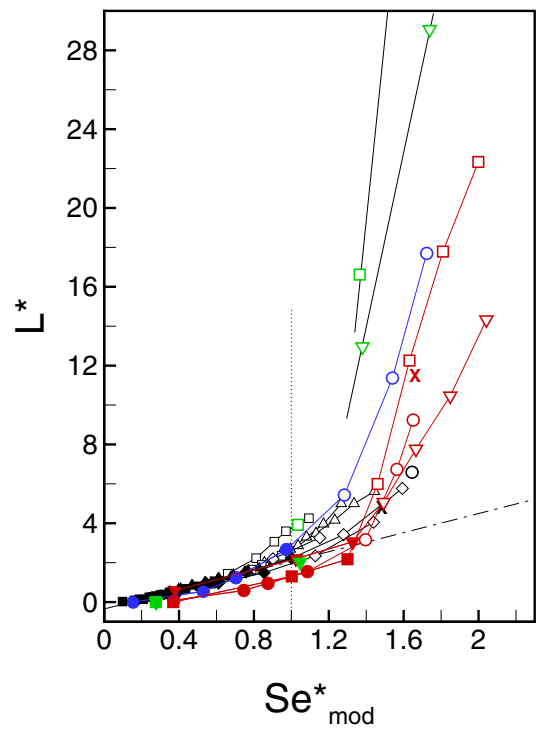

(b)

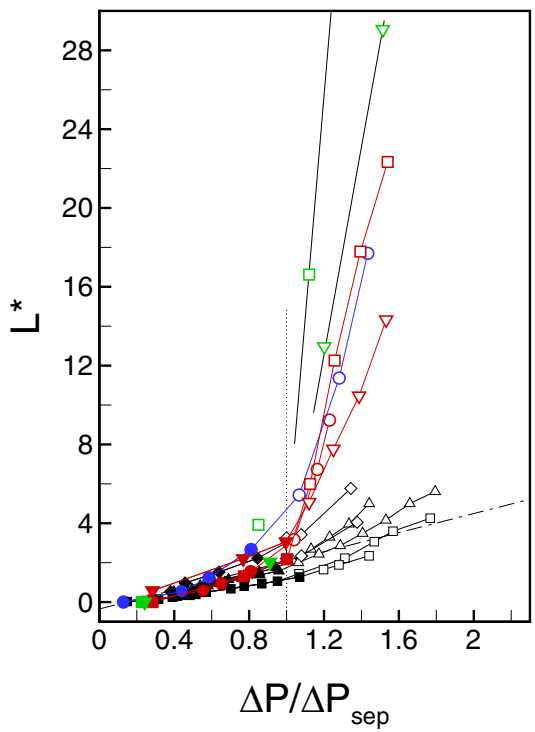

FIG. 7. Supersonic and hypersonic scaled STBLI data plotted together with $L^{*}$ versus $S e_{\bmod }^{*}$ (a) compared to $L^{*}$ versus $\Delta P / \Delta P_{\text {sep }}$ (b). Symbols are as in Fig. 3 for supersonic data and Fig. 6 for hypersonic data. Filled symbols are incipiently separated and open symbols are fully separated.

In comparing Figs. 3(a) and 3(b) and also Figs. 7(a) and 7(b) we propose that the modified separation scaling derived in Sec. III is the appropriate separation-length-to-shock-strength scaling for the incipient separation regime. For the incipient interactions, the boundary layer separates when the pressure jump across the shock structure is sufficient to halt the momentum of the incoming boundary layer and so $\Delta P$ scales by $q_{\mathrm{e}}$. Increasing the wall temperature will increasingly skew the distribution of momentum towards the edge of the boundary layer and so we see that heated interactions produce larger separation than an adiabatic case at the same deflection angle. The opposite effect occurs when the wall is cooled. The skin friction ratio correction on $k_{\text {mod }}$ was shown in Sec. III to account for the variation in separation length caused by the wall temperature condition. Also by the current data compilation, the incipient separation data collapse appears to be independent of the geometry and agrees for all compression ramp, reflected shock, and axisymmetric flare data. In contrast, the separated interactions follow a different trend and a different Reynolds number dependence.

There is much evidence, both experimental [26,27] and computational [26,28-30], that supersonic separated STBLIs contain certain inviscid vortical structures (IVSs) that are aligned with the streamwise direction and mix the inviscid high momentum fluid with the near wall separated flow. These IVSs have also been observed in hypersonic separated interactions, both turbulent $[31,32]$ and laminar [33-35]. Conceptually similar to the inviscid Görtler vortices occurring in subsonic laminar boundary layers over concave surfaces, the IVSs originate from the elevated streamline curvature at the separation point and extend downstream with the vortex cores oriented in the streamwise direction. Alternatively, a second theory regarding the origin of the IVS in laminar flow has been formulated that these large vortical structures are a product of the 3D structure of the separated flow [35], and it remains undetermined if this applies to turbulent boundary layer interactions. The wall-normal extent of these vortices is larger than the boundary layer thickness. They produce significant mixing between the inviscid flow and viscous boundary layer and carry with them a large amount of energy and momentum. It is argued by Martin et al. [36] and Martin and Helm [37] that the circulation of the IVS is such that it depletes the separation bubble, thus tending to reduce the 
(a)

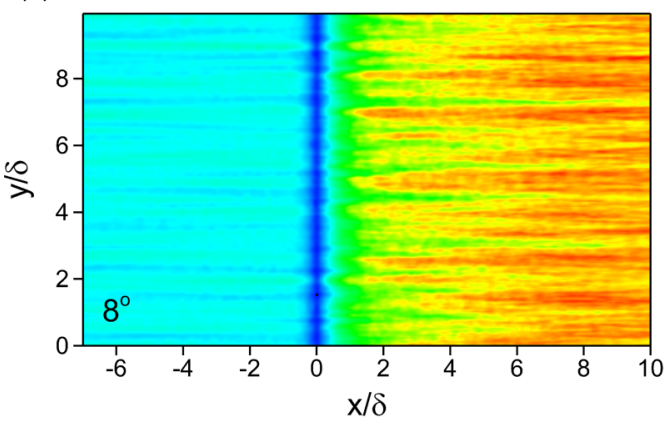

(b)

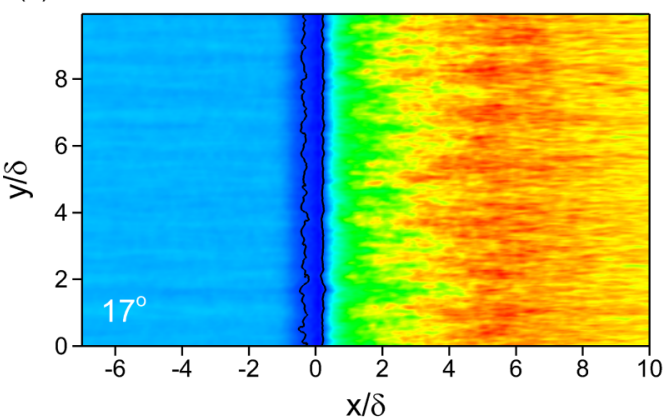

(c)

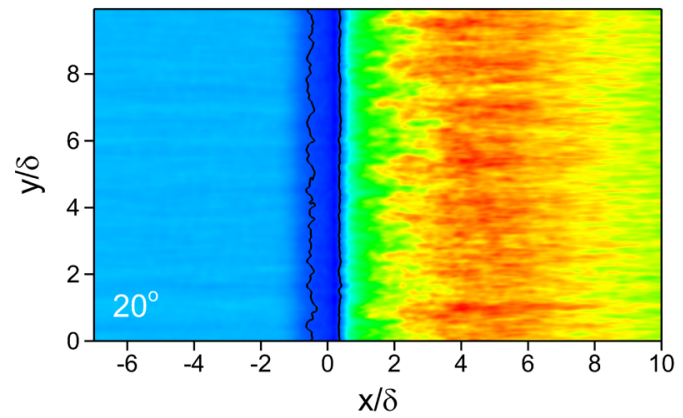

(e)

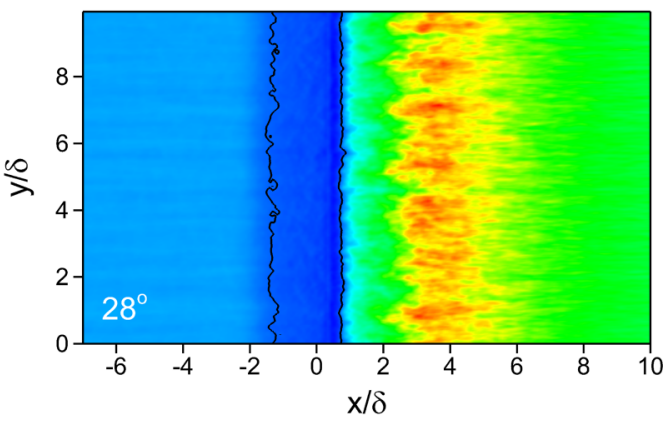

(f)

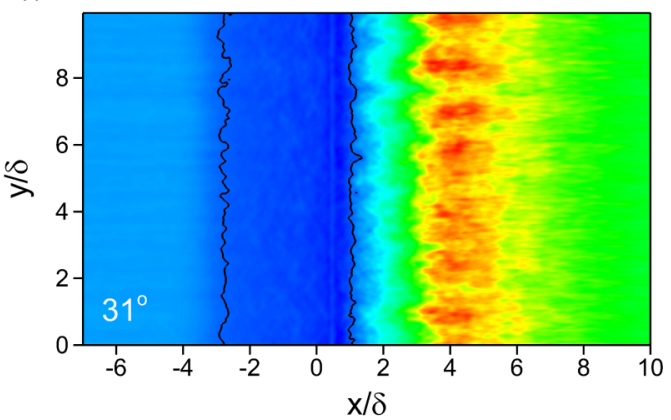

(g)

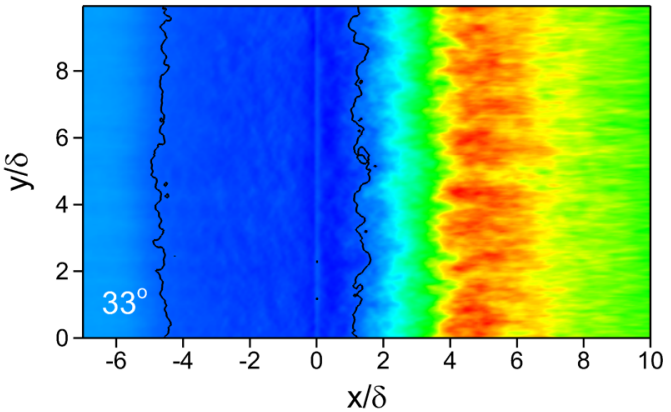

(d)

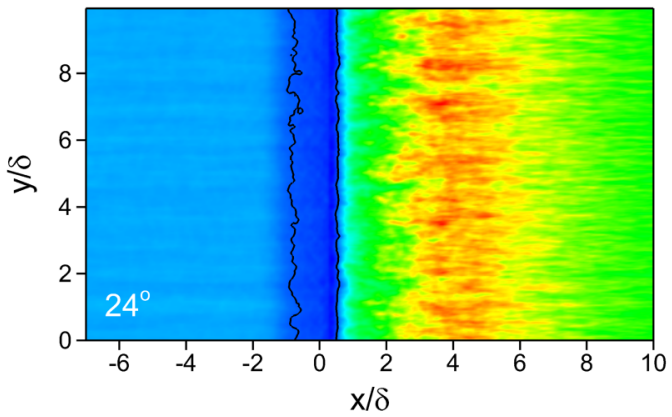

FIG. 8. 2D contours of time-averaged streamwise skin friction for various angles of the M7 LES. 

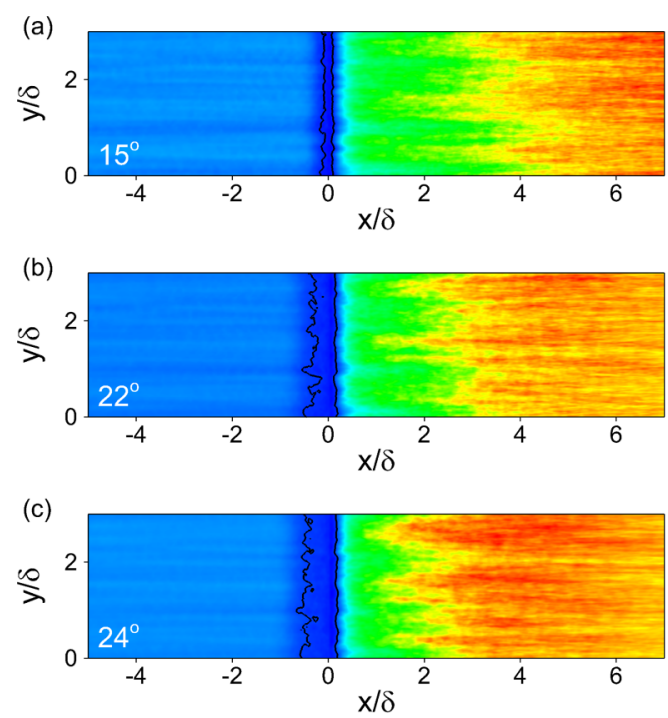
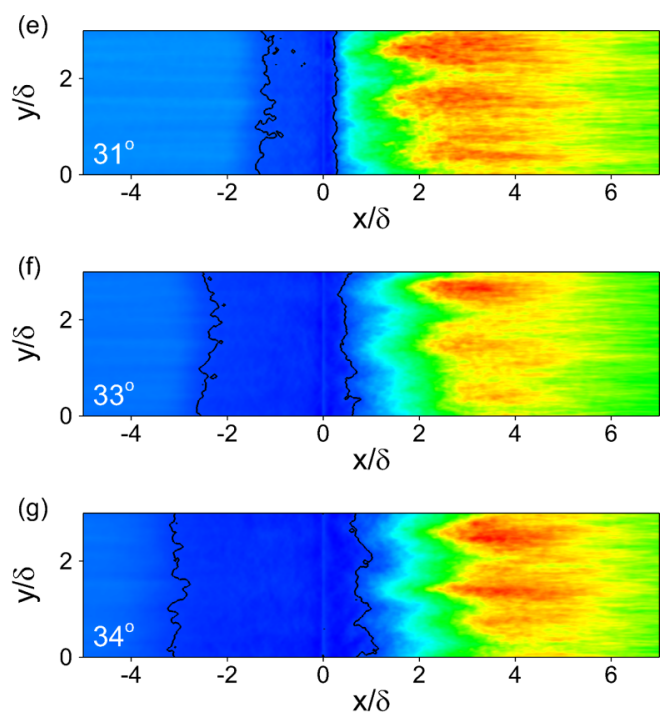

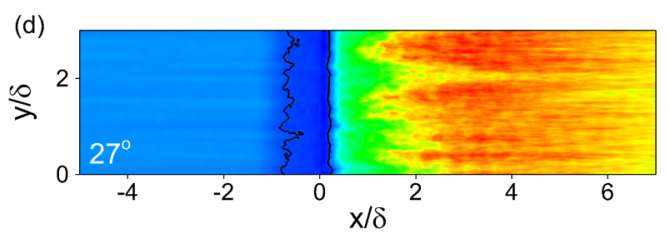

FIG. 9. 2D contours of time-averaged streamwise skin friction for various angles of the M10 LES.

separation length. It can therefore be argued that the strength of the IVS circulation is a significant factor in determining the average separation length of the interaction.

With the current limited data compilation and the limited knowledge of the factors affecting the dynamics of the IVS, it is not possible to propose a scaling for the fully separated STBLI regime. We observe, however, that the data trends can be reconciled with the existence of IVS. Shown by the data in Fig. 6, there is an obvious dependence of $L^{*}$ on Reynolds number when comparing between M10-Elf1 and M10-Elf2 and also between M10-Col1 and M10-Col2. The $L^{*}$ is significantly reduced in the lower Reynolds number data. Notice that an increase in the Reynolds number of the incoming boundary layer results in an increase in the turbulent mixing and energy in the incoming boundary layer flow, which in turn hinder the development of the IVS resulting in weaker circulation of the vortices rendering them less effective in depleting the separation bubble. This is consistent with the fact that the mean separation length increases with increasing Reynolds number for a given $M_{\mathrm{e}}$ and $\phi$ as the data of Elfstrom and Coleman show. In the case of M10-34, the IVS are confined by the narrow grid resulting in a stronger instability with stronger circulation. The result is again a smaller separation length in M10-34 compared to M10-34w for which there is no such constriction. For the cylinder-flare data compared to the compression ramp data the spanwise relief effects from the increasing flare radius downstream from attachment significantly weakens the strength of the IVS and the separation length increases dramatically.

Arguments for the physical mechanisms that could be at play in determining the separation length scaling for the two separation regimes, incipient and full separation, have been discussed. The footprint of the IVS presents itself as spanwise-repeating "cells" in the contours of skin friction downstream from reattachment $[26,27,29,32]$. In Fig. 8 are plotted contours of streamwise skin friction on the streamwise-spanwise floor plane for the M7 interactions at the various compression angles. The same quantity is shown in Fig. 9 for the M10 interactions. The skin friction is normalized 
by the maximum of $C_{f}$ as realized in the given ramp angle. Plotting in this way highlights the skin friction pattern on the ramp surface. The footprint of IVS is apparent with spanwise periodicity approximately $2 \delta$. These appear as bright red "spots" in the contours of Fig. 8 and 9 with a typical width of approximately $1 \delta$ per spot. Only the case that is fully attached in the mean sense M7-08 has no noticeable imprint of the IVS. Slight traces of the IVS structure can be made out even in the very mildly separated cases M10-15 in Fig. 9(a) and M7-17 in Fig. 8(b). For the conditions we considered, the data contours suggest that even the slightest reverse flow in the mean sense is enough to produce the IVS. Thus, the intermittently separated flow reflects IVS but they are weak due to a small separation bubble with shallower streamline curvature. Under these conditions, we postulate that the length of the separation bubble is minimally affected by the presence of the IVS, and the momentum of the incoming boundary layer has the greater influence on establishing the mean separation length. Thus the scaling by $S e_{\text {mod }}^{*}$ holds. As the flow deflection angle increases, the streamline curvature at separation also increases producing stronger IVS.

\section{ACKNOWLEDGMENTS}

This work is supported in part by the Air Force Office of Scientific Research, Award No. FA955017-1-0104, and the Air Force Testing and Evaluation Division, Award No. FA9550-10-1-0535 with the Center for Testing Excellence at the University of Maryland and the Arnold Engineering Development Center.

[1] L. J. Souverein, P. G. Bakker, and P. Dupont, A scaling analysis for turbulent shock-wave/boundary-layer interactions, J. Fluid Mech. 714, 505 (2013).

[2] V. Jaunet, J. F Debieve, and P. Dupont, Length scales and time scales of a heated shock-wave/boundarylayer interaction, AIAA J. 52, 2524 (2014).

[3] D. R. Chapman, D. M. Kuehn, and H. K. Larson, Investigation of separated flows in supersonic and subsonic streams with emphasis on the effect of transition, NACA, Report No. 1356, 1958.

[4] F. W. Spaid and J. C. Frishett, Incipient separation of a supersonic, turbulent boundary layer, including effect of heat transfer, AIAA J. 10, 915 (1972).

[5] P. S. Volpiani, M. Bernardini, and J. Larsson, Effects of a nonadiabatic wall on supersonic shock/boundary-layer interactions, Phys. Rev. Fluids 3, 083401 (2018).

[6] G. M. Elfstrom, Turbulent hypersonic flow at a wedge-compression corner, J. Fluid Mech. 53, 113 (1972).

[7] G. T. Coleman, Hypersonic turbulent boundary layer studies, Ph.D. thesis, University of London, 1973.

[8] J. Brooks, A. K. Gupta, C. Helm, M. P. Martin, M. Smith, and E. Marineau, Mach 10 PIV flow field measurements of a turbulent boundary layer and shock turbulent boundary layer interaction, in 33rd AIAA Aerodynamic Measurement Technology and Ground Testing Conference (AIAA, Reston, VA, 2017), AIAA Paper No. 2017-3325 (2017).

[9] J. L. Sims, Tables for supersonic flow around right circular cones at zero angle of attack, NASA Technical Report, NASA-SP-3004, 1964.

[10] P. Dupont, SBD originally reported a mild dependence on Reynolds number so that $k=3$ for $\operatorname{Re}_{\theta} \leqslant$ $1 \times 10^{4}$. It was later determined that this shift in $k$ was a result of three-dimensional effects in the experimental data. After correction, $k$ was found to be 2.5 also for the lower Reynolds number data (private communication).

[11] E. J. Hopkins and M. Inouye, An evaluation of theories for predicting turbulent skin friction and heat transfer on flat plates at supersonic and hypersonic mach numbers, AIAA J. 9, 993 (1971).

[12] S. Priebe and M. P. Martin, Low-frequency unsteadiness in shock wave-turbulent boundary layer interaction, J. Fluid Mech. 699, 1 (2012).

[13] B. Ganapathisubramani, N. T. Clemens, and D. S. Dolling, Effects of upstream boundary layer on the unsteadinessof shock-induced separation, J. Fluid Mech. 585, 369 (2007). 
[14] M. P. Martin, E. M. Taylor, M. Wu, and V. G. Weirs, A bandwidth-optimized WENO scheme for the direct numerical simulation of compressible turbulence, J. Comput. Phys. 220, 270 (2006).

[15] E. M. Taylor, M. Wu, and M. P. Martin, Optimization of nonlinear error for weighted essentially nonoscillatory methods in direct numerical simulations of compressible turbulence, J. Comput. Phys. 223, 384 (2007).

[16] J. H. Williamson, Low-storage Runge-Kutta schemes, J. Comput. Phys. 35, 48 (1980).

[17] M. P. Martin, U. Piomelli, and G. V. Candler, Subgrid-scale models for compressible large-eddy simulations, Theor. Comput. Fluid Dyn. 13, 361 (2000).

[18] D. Knight, G. Zhou, N. Okong'o, and V. Shukla, Compressible large eddy simulation using unstructured grids, in 36th AIAA Aerospace Science Meeting and Exhibit (AIAA, Reston, VA, 1998), AIAA Paper No. 1998-0535.

[19] C. M. Helm and M. P. Martin, Large eddy simulation of two separated hypersonic shock/turbulent boundary layer interactions (unpublished).

[20] P. Dupont, C. Haddad, and J. F. Debiève, Space and time organization in a shock induced separated boundary layer, J. Fluid Mech. 559, 255 (2006).

[21] C. Helm and M. P. Martin, New LES of a hypersonic shock/turbulent boundary layer interaction, in 54th Aerospace Science Meeting (AIAA, Reston, VA, 2016), AIAA Paper No. 2016-0346.

[22] G. M. Elfstrom, Turbulent separation in hypersonic flow, Ph.D. thesis, University of London, 1971.

[23] E. R van Driest, The problem of aerodynamic heating, Aeronaut. Eng. Rev. 15, 26 (1956).

[24] L. Duan, I. Beekman, and M. P. Martin, Direct numerical simulation of hypersonic turbulent boundary layers. Part 2. Effect of wall temperature, J. Fluid Mech. 655, 419 (2010).

[25] L. Duan, I. Beekman, and M. P. Martin, Direct numerical simulation of hypersonic turbulent boundary layers. Part 3. Effect of Mach number, J. Fluid Mech. 672, 245 (2011).

[26] M. S. Loginov, N. A. Adams, and A. A. Zheltovodov, Large-eddy simulation of shock-wave/turbulentboundary-layer interaction, J. Fluid Mech. 565, 135 (2006).

[27] G. S. Settles, T. J. Fitzpatrick, and S. M. Bogdonoff, Detailed study of attached and separated compression corner flowfields in high Reynolds number supersonic flow, AIAA J. 17, 579 (1979).

[28] S. Priebe, J. H. Tu, C. W. Rowley, and M. P. Martin, Low-frequency dynamics in a shock-induced separated flow, J. Fluid Mech. 807, 441 (2016).

[29] D. M. Dawson and S. K. Lele, Large eddy simulation of a three-dimensional compression ramp shockturbulent boundary layer interaction, in 53rd AIAA Aerospace Sciences Meeting (AIAA, Reston, VA, 2015), AIAA Paper No. 2015-1518.

[30] M. Grilli, S. Hickel, and N. A. Adams, Large-eddy simulation of a supersonic turbulent boundary layer over a compression-expansion ramp, Int. J. Heat Fluid Flow 42, 79 (2013).

[31] J. R. Edwards, J.-I. Choi, and J. A. Boles, Large-eddy/Reynolds-averaged Navier-Stokes simulation of a Mach 5 compression-corner interaction, AIAA J. 46, 977 (2008).

[32] J. Trichilo, C. M. Helm, and M. P. Martin, Persistence of a centrifugal instability in shock-separated flows at Mach 3 through 10, in AIAA Scitech 2019 Forum (AIAA, Reston, VA, 2019), AIAA Paper No. 2019-1130.

[33] S. Navarro-Martinez and O. R. Tutty, Numerical simulation of görtler vortices in hypersonic compression ramps, Comput. Fluids 34, 225 (2005).

[34] S. Cao, I. Klioutchnikov, and H. Olivier, Görtler vortices in hypersonic flow on compression ramps, AIAA J. 57, 3874 (2019).

[35] N. Hildebrand, A. Dwivedi, J. W. Nichols, M. R. Javonović, and G. V. Candler, Simulation and stability analysis of oblique shock-wave/boundary-layer interactions at mMach 5.92, Phys. Rev. Fluids 3, 013906 (2018).

[36] M. P. Martin, S. Priebe, and C. M Helm, Upstream and downstream influence on shock and turbulent boundary layer instability, in 46th AIAA Fluid Dynamics Conference (AIAA, Reston, VA, 2016), AIAA Paper No. 2016-3341.

[37] M. P. Martin and C. M Helm, The role of inviscid centrifugal instability vortices in shock-separated boundary layers (unpublished). 